\title{
ON THE RADIUS OF CONVEXITY AND BOUNDARY DISTORTION OF SCHLICHT FUNCTIONS $\left(^{1}\right)$
}

\author{
BY \\ DAVID E. TEPPER
}

\begin{abstract}
Let $w=f(z)=z+\sum_{n=2}^{\infty} a_{n} z^{n}$ be regular and univalent for $|z|<1$ and map $|z|<1$ onto a region which is starlike with respect to $w=0$. If $r_{0}$ denotes the radius of convexity of $w=f(z), d_{0}=\min |f(z)|$ for $|z|=r_{0}$, and $d^{*}=\inf |\beta|$ for $f(z) \neq \beta$, then it has been conjectured that $d_{0} / d^{*} \geqq 2 / 3$. It is shown here that $d_{0} / d^{*} \geqq 0.343 \ldots$, which improves the old estimate $d_{0} / d^{*} \geqq 0.268 \ldots$. In addition, sharp estimates for $r_{0}$ are given which depend on the value of $\left|a_{2}\right|$.
\end{abstract}

1. Introduction. It is shown in [2] that if $w=f(z)=z+\sum_{n=2}^{\infty} a_{n} z^{n}$ is regular and univalent for $|z|<1$, then there is a positive number $r_{0}$, such that $w=f(z)$ maps $|z| \leqq r_{0}$. onto a convex region. Furthermore, it is shown that $r_{0} \geqq 2-\sqrt{ } 3$ for all functions $w=f(z)$ which are regular and univalent for $|z|<1$. From this we see that associated with every function $w=f(z)=z+\sum_{n=2}^{\infty} a_{n} z^{n}$ regular and univalent for $|z|<1$, there is a radius of convexity $r_{0}$ which is the largest number such that $w=f(z)$ maps $|z| \leqq r_{0}$ onto a convex region and need not map $|z| \leqq r$ onto a convex region when $r>r_{0}$.

In this paper the following question is considered: Let $w=f(z)=z+\sum_{n=2}^{\infty} a_{n} z^{n}$ be regular and univalent for $|z|<1$ and map $|z|<1$ onto a region which is starlike with respect to $w=0$. If $r_{0}$ denotes the radius of convexity of $w=f(z), d_{0}=$ $\min _{|z|=r_{0}}|f(z)|$, and $d^{*}=\inf |\beta|, f(z) \neq \beta$, then in [9] it is conjectured that $d_{0} / d^{*} \geqq 2 / 3$. This lower limit cannot be improved since it is attained for the function $w=f(z)=z(1-z)^{-2}$. In this paper the conjecture is demonstrated for certain classes of functions, while for other functions lower estimates for $d_{0} / d^{*}$ are found. Presently,the best estimate for all starlike maps is $d_{0} / d^{*}>2-\sqrt{ } 3=.268 \ldots$, see [9].

In order to obtain estimates for $d_{0} / d^{*}$, we study how the second coefficient in the expansion of the function $w=f(z)=z+\sum_{n=2}^{\infty} a_{n} z^{n}$ regular and univalent for $|z|<1$ affects certain properties of this function. This type of problem was first studied by Gronwall in [4]. In this paper we generalize results of Finkelstein [1] to the class of

Presented to the Society, January 25, 1969; received by the editors April 15, 1969 and, in revised form, November 10, 1969.

AMS Subject Classifications. Primary 3042, 3043.

Key Words and Phrases. Schlicht functions, convex and starlike functions, radius of convexity.

(1) This paper is part of the author's doctoral dissertation at Temple University. The author wishes to thank Professor Albert Schild for his guidance and encouragement during the preparation of this paper. 
functions $w=f(z)=z+\sum_{n=2}^{\infty} a_{n} z^{n}$ which are starlike of order $\alpha$. These functions, which are characterized by $\operatorname{Re}\left(z f^{\prime}(z) / f(z)\right) \geqq \alpha$ for $0 \leqq \alpha \leqq 1$, were first introduced in [8]. Furthermore we give sharp lower bounds for the radius of convexity which depend on the second coefficient in the expansion of $w=f(z)=z+\sum_{n=2}^{\infty} a_{n} z^{n}$ which is regular and univalent for $|z|<1$ and maps $|z|<1$ onto a region which is starlike with respect to $w=0$. Using these estimates we show $d_{0} / d^{*} \geqq .343 \ldots$ The method used to obtain this estimate is then generalized to the class of functions which has $p$-fold rotational symmetry. It is here that the conjecture for $d_{0} / d^{*}$ is demonstrated for certain classes of functions.

Notation. Let $U$ denote the class of functions $w=f(z)=z+\sum_{n=2}^{\infty} a_{n} z^{n}$ which are regular and univalent for $|z|<1$. Let St denote the class of functions $w=f(z) \in U$ which map $|z|<1$ onto a starlike region with respect to $w=0$. Finally, let $\mathrm{St}_{\alpha}$ denote the class of functions $w=f(z) \in U$ which are starlike of order $\alpha$, for $0 \leqq \alpha \leqq 1$. It is well known that $\mathrm{St}_{0}=\mathrm{St}$.

2. Preliminaries. In this section we prove two lemmas concerning functions which have positive real part.

Lemma 1. If $P(z)=1+b z+\sum_{n=2}^{\infty} b_{n} z^{n}$ is regular and has $\operatorname{Re} P(z)>0$ for $|z|<1$, then

$$
\operatorname{Re} P(z) \geqq \frac{1-|z|^{2}}{1+b|z|+|z|^{2}}
$$

where $b \geqq 0$. Furthermore, this result is sharp for each value of $b, 0 \leqq b \leqq 2$ by considering the functions $P_{b}(z)=\left(1-z^{2}\right)\left(1-b z+z^{2}\right)^{-1}$.

Proof. Since $\operatorname{Re} P(z)>0$ and $P(0)=1$, the function $P(z)$ is subordinate to the function $(1+z)(1-z)^{-1}$; see [5, p. 228]. Therefore, there exists a function $h(z)$ which is regular for $|z|<1$ with $h(0)=0$ and $|h(z)|<1$ such that:

$$
P(z)=\frac{1+h(z)}{1-h(z)}=1+b z+\sum_{n=2}^{\infty} b_{n} z^{n} .
$$

A direct computation gives $h(z)=b z / 2+\cdots$. Therefore, by a generalized form of Schwarz's Lemma [5, p. 167],

$$
|h(z)| \leqq|z| \frac{|z|+b 2^{-1}}{1+b 2^{-1}|z|}=|z| \frac{2|z|+b}{2+b|z|} .
$$

Another direct computation shows

$$
\operatorname{Re} P(z)=\frac{1-|h(z)|^{2}}{|1-h(z)|^{2}} \geqq \frac{1-|h(z)|}{1+|h(z)|}
$$

since the right-hand side of (4) is monotone decreasing with respect to $|h(z)|$, applying (3) to (4) we obtain

$$
\operatorname{Re} P(z) \geqq \frac{1-|z|^{2}}{1+b|z|+|z|^{2}} .
$$


A direct computation shows sharpness.

LEMMA 2. If

$$
P(z)=1+b z+\sum_{n=2}^{\infty} b_{n} z^{n}
$$

is regular and has $\operatorname{Re} P(z)>0$ for $|z|<1$, then

$$
|P(z)| \leqq \frac{1+b|z|+|z|^{2}}{1-|z|^{2}},
$$

where $b \geqq 0$. Furthermore, this result is sharp for each value of $b, 0 \leqq b \leqq 2$, by considering the functions $P^{b}(z)=\left(1-z^{2}\right)\left(1-b z+z^{2}\right)^{-1}$.

Proof. Consider the function $Q(z)=P(-z)^{-1}$. Since $Q(z)$ obeys the hypothesis of Lemma 1, we have

$$
\frac{1}{|P(z)|}=|Q(-z)| \geqq \operatorname{Re} Q(-z) \geqq \frac{1-|z|^{2}}{1+b|z|+|z|^{2}} .
$$

A direct computation shows sharpness.

3. Estimates for the class $\mathrm{St}_{\alpha}$. In this section we give estimates for the function $w=f(z)=z+\sum_{n=2}^{\infty} a_{n} z^{n} \in \mathrm{St}_{\alpha}$. For notational convenience we will write $a_{2}=a$. It is no loss of generality to suppose $a \geqq 0$. If this is not the case, then consider the function $w=e^{i \theta} f\left(e^{-i \theta} z\right)$ where $\theta=\arg a$.

THEOREM 1. If $w=f(z) \in \mathrm{St}_{\alpha}$, then

$$
\operatorname{Re} \frac{z f^{\prime}(z)}{f(z)} \geqq \frac{(1-\alpha)+a \alpha|z|+(1-\alpha)(2 \alpha-1)|z|^{2}}{(1-\alpha)+a|z|+(1-\alpha)|z|^{2}} .
$$

Proof. By the principle of subordination, there exists a function $P(z)=z+b z$ $+\sum_{n=2}^{\infty} b_{n} z^{n}$ which is regular and has $\operatorname{Re} P(z)>0$ for $|z|<1$, such that

$$
z f^{\prime}(z) / f(z)=(1-\alpha) P(z)+\alpha ;
$$

see [5, p. 228]. Furthermore, a direct computation shows

$$
z f^{\prime}(z) / f(z)=1+a z+\cdots
$$

Equating coefficients of $z$ in (9), we have $b=a(1-\alpha)^{-1}$. Therefore, by Lemma 1 we have

$$
\operatorname{Re} P(z) \geqq \frac{1-|z|^{2}}{1-a(1-\alpha)^{-1}|z|+|z|^{2}}
$$

Using (11) on (9), we obtain (8).

THEOREM 2. If $w=f(z) \in \mathrm{St}_{\alpha}$, then

$$
|f(z)| \geqq|z|\left[\frac{1-\alpha}{(1-\alpha)+a|z|+(1-\alpha)|z|^{2}}\right]^{1-\alpha} \text {. }
$$


Proof. If $z=r e^{i \theta}$, then

$$
\frac{\partial}{\partial r} \log \left|\frac{f(z)}{z}\right|=\operatorname{Re} \frac{z f^{\prime}(z)}{f(z)}-1 .
$$

Applying Theorem 1 to (13), we obtain

$$
\frac{\partial}{\partial r} \log \left|\frac{f(z)}{z}\right| \geqq-(1-\alpha) \frac{a+2(1-\alpha) r}{(1-\alpha)+a r+(1-\alpha) r^{2}} .
$$

Integrating from $r=0$ to $r=|z|<1$, after taking exponents, we obtain inequality (12).

THEOREM 3. If $w=f(z) \in \mathrm{St}_{\alpha}$, then

$$
\left|f^{\prime}(z)\right| \geqq(1-\alpha)^{1-\alpha}\left\{\frac{(1-\alpha)+a \alpha|z|+(2 \alpha-1)(1-\alpha)|z|^{2}}{\left[(1-\alpha)+a|z|+(1-\alpha)|z|^{2}\right]^{2-\alpha}}\right\}
$$

Proof. The result follows by applying inequality (12) to inequality (8).

A direct computation shows that

$$
f_{\alpha, a}(z)=z\left[\frac{1-\alpha}{(1-\alpha)-a z+(1-\alpha) z^{2}}\right]^{1-\alpha}
$$

gives extremal functions for Theorems 1, 2, and 3. Using Lemma 2 in a similar manner in which Lemma 1 was used, the following theorem may be proven.

THEOREM 4. If $w=f(z) \in \mathrm{St}_{\alpha}$, then

$$
\begin{gathered}
\left|\frac{z f^{\prime}(z)}{f(z)}\right| \leqq \frac{1+a|z|+(1-2 \alpha)|z|^{2}}{1-|z|^{2}}, \\
|f(z)| \leqq|z|\left[\frac{1+|z|}{1-|z|}\right]^{a / 2} \frac{1}{\left(1-|z|^{2}\right)^{1-\alpha}}, \\
\left|f^{\prime}(z)\right| \leqq\left[\frac{1+|z|}{1-|z|}\right]^{a / 2} \frac{1+a|z|+(1-2 \alpha)|z|^{2}}{\left(1-|z|^{2}\right)^{2-\alpha}} .
\end{gathered}
$$

A direct computation shows that

$$
F_{\alpha, a}(z)=z\left[\frac{1+z}{1-z}\right]^{a / 2} \frac{1}{\left(1-z^{2}\right)^{1-\alpha}}
$$

gives extremal functions for Theorem 4.

Let $K$ denote the class of functions $w=f(z) \in U$ which map $|z|<1$ onto a convex region. Clearly, $K \subset \mathrm{St}$. In [13], Strohhäcker proved $K \subset \mathrm{St}_{1 / 2}$. In [10], Schild proved that

$$
|z|(1+|z|)^{-1} \leqq|f(z)| \leqq|z|(1-|z|)^{-1}
$$

when $w=f(z) \in \mathrm{St}_{1 / 2}$. Since the extremal function for (18), which is $w=f(z)$ $=z(1-z)^{-1}$ maps $|z|<1$ onto a convex region, we see that the same estimate for 
$|f(z)|$ holds for the class $K$ as the class $\mathrm{St}_{1 / 2}$. However, in [4], Gronwall proved that

$$
\begin{aligned}
|f(z)| & \geqq \frac{1}{\sqrt{ }\left(1-a^{2}\right)} \operatorname{Arctan} \frac{|z| \sqrt{ }\left(1-a^{2}\right)}{1+a|z|}, \quad \text { for } 0 \leqq a<1, \\
& \geqq \frac{|z|}{1+|z|}, \quad \text { for } a=1,
\end{aligned}
$$

when $w=f(z) \in K$. It is interesting to notice that

$$
\frac{1}{\sqrt{ }\left(1-a^{2}\right)} \operatorname{Arctan} \frac{|z| \sqrt{ }\left(1-a^{2}\right)}{1+a|z|}>\frac{|z|}{\sqrt{ }\left(1+2 a|z|+|z|^{2}\right)}
$$

when $a \neq 1$.

4. Radius of convexity estimates. The following two lemmas enable us to give sharp estimates for $r_{0}$ when $w=f(z) \in$ St has a preassigned second coefficient. Again we will write $a_{2}=a$ and we will assume $a \geqq 0$.

LEMMA 3. If $P(z)=1+b z+\sum_{n=3}^{\infty} b_{n} z^{n}$ is regular and has $\operatorname{Re} P(z)>0$ for $|z|<1$, then

$$
\left|z \frac{P^{\prime}(z)}{P(z)}\right| \leqq \frac{|z|}{1-|z|^{2}} \frac{b|z|^{2}+4|z|+b}{|z|^{2}+b|z|+1}
$$

where $b \geqq 0$.

Proof. As was shown in Lemma 1 , there exists a function $h(z)=b z / 2+\cdots$ regular for $|z|<1$ with $|h(z)|<1$, such that $P(z)=(1+h(z)) /(1-h(z))$. Furthermore, since $h(0)=0$ and $|h(z)|<1$, there exists a function $\phi(z)$ regular for $|z|<1$, such that $h(z)=z \phi(z)$. Using this we obtain $P(z)=(1+z \phi(z)) /(1-z \phi(z))$. Taking the logarithmic derivatives of both sides we have

$$
\frac{P^{\prime}(z)}{P(z)}=2 \frac{\phi(z)+z \phi^{\prime}(z)}{1-z^{2} \phi^{2}(z)}
$$

Using the triangle inequality, we have

$$
\left|\frac{P^{\prime}(z)}{P(z)}\right| \leqq 2 \frac{|\phi(z)|+|z|\left|\phi^{\prime}(z)\right|}{1-|z|^{2}|\phi(z)|^{2}}
$$

In [5], it is proven that

$$
\left|\phi^{\prime}(z)\right| \leqq \frac{1-|\phi(z)|^{2}}{1-|z|^{2}}
$$

Since the right-hand side of (20) is monotone increasing with respect to $\left|\phi^{\prime}(z)\right|$, by substituting (21) into (20) we obtain

$$
\left|\frac{P^{\prime}(z)}{P(z)}\right| \leqq \frac{2}{1-|z|^{2}}\left[\frac{|\phi(z)|\left(1-|z|^{2}\right)+|z|\left(1-|\phi(z)|^{2}\right)}{1-|z|^{2}|\phi(z)|^{2}}\right] .
$$


We wish to show the expression in square brackets in (22) is monotone increasing with respect to $|\phi(z)|$. To do this consider

$$
g(x)=\frac{x\left(1-r^{2}\right)+r\left(1-x^{2}\right)}{1-r^{2} x^{2}}
$$

where $0 \leqq x=|\phi(z)| \leqq 1$ and $0 \leqq r=|z|<1$. Differentiating (23) we obtain

$$
g^{\prime}(x)=\frac{\left(1-r^{2}\right)(1-r x)^{2}}{\left(1-r^{2} x^{2}\right)^{2}} \geqq 0
$$

because $0 \leqq r<1$ and $0 \leqq x \leqq 1$.

Furthermore, by inequality (3) we have

$$
|\phi(z)|=\left|\frac{h(z)}{z}\right| \leqq \frac{2|z|+b}{2+b|z|} .
$$

Since the expression in square brackets in (22) is monotone increasing with respect to $|\phi(z)|$, we obtain

$$
\begin{aligned}
\left|\frac{P^{\prime}(z)}{P(z)}\right| & \leqq \frac{2}{1-|z|^{2}} \frac{\left(\frac{2|z|+b}{2+b|z|}\right)\left(1-|z|^{2}\right)+|z|\left[1-\left(\frac{2|z|+b}{2+b|z|}\right)^{2}\right]}{1-|z|^{2}\left(\frac{2|z|+b}{2+b|z|}\right)^{2}} \\
& =\frac{1}{1-|z|^{2}} \frac{\left(1-|z|^{2}\right)\left(b|z|^{2}+4|z|+b\right)}{\left(1-|z|^{2}\right)\left(|z|^{2}+b|z|+1\right)}
\end{aligned}
$$

which completes the proof.

LEMMA 4. If $w=f(z) \in \mathrm{St}$, then

$$
\operatorname{Re} \frac{z f^{\prime \prime}(z)}{f^{\prime}(z)}+1 \geqq \frac{1}{1-|z|^{2}} \frac{1-a|z|-6|z|^{2}-a|z|^{3}+|z|^{4}}{1+a|z|+|z|^{2}} .
$$

Proof. If

$$
P(z)=z f^{\prime}(z) / f^{\prime}(z)=1+a z+\cdots,
$$

then since $w=f(z) \in \mathrm{St}$, Theorem 1 gives

$$
\operatorname{Re} P(z) \geqq \frac{1-|z|^{2}}{1+a|z|+|z|^{2}}
$$

Direct computation gives

$$
z f^{\prime \prime}(z) / f^{\prime}(z)+1=P(z)+z P^{\prime}(z) / P(z) .
$$

Therefore, applying (25) and (19) to this equation we obtain

$$
\begin{aligned}
\operatorname{Re} \frac{z f^{\prime \prime}(z)}{f^{\prime}(z)}+1 & \geqq \operatorname{Re} P(z)-\left|z \frac{P^{\prime}(z)}{P(z)}\right| \\
& \geqq \frac{1}{1-|z|^{2}} \frac{1-a|z|-6|z|^{2}-a|z|^{3}+|z|^{4}}{1+a|z|+|z|^{2}}
\end{aligned}
$$

which completes the proof of Lemma 4. 
We are now ready to give estimates for the radius of convexity for functions in St.

THEOREM 5. If $w=f(z) \in \mathrm{St}$ and has the radius convexity $r_{0}$, then

$$
r_{0} \geqq r_{0}(a)=\frac{a+\sqrt{ }\left(a^{2}+32\right)-\sqrt{ }\left[2 a^{2}+2 a \sqrt{ }\left(a^{2}+32\right)+16\right]}{4} .
$$

This estimate is sharp for each $a, 0 \leqq a \leqq 2$ by considering the functions $f_{a}(z)=$ $z /\left(1-a z+z^{2}\right)$.

Proof. By [2] we have that $w=f(z) \in U$ maps $|z| \leqq r$ onto a convex region if and only if $\operatorname{Re}\left(z f^{\prime \prime}(z) / f^{\prime}(z)\right)+1 \geqq 0$ for $|z| \leqq r$. Therefore by Lemma $4, w=f(z)$ will map $|z| \leqq r$ onto a convex region if

$$
\operatorname{Re} \frac{z f^{\prime \prime}(z)}{f^{\prime}(z)}+1 \geqq \frac{1}{1-|z|^{2}} \frac{1-a|z|-6|z|^{2}-a|z|^{3}+|z|^{4}}{1+a|z|+|z|^{2}} \geqq 0 .
$$

for $|z|<r$. Therefore, the radius of convexity of $w=f(z)$ is greater than or equal to the least positive root of

$$
q_{a}(r)=1-a r-6 r^{2}-a r^{3}+r^{4}=0,
$$

which is exactly $r_{0}(a)$. A direct computation verifies sharpness.

5. Estimates for $d_{0} / d^{*}$. In this section we prove $d_{0} / d^{*}>.343 \ldots$ In order to obtain this estimate, we need the following lemmas.

Lemma 5. If $w=f(z) \in \mathrm{St}$, then

$$
\begin{array}{rlrl}
d^{*} & \leqq 2 /(a+2), & & 0 \leqq a \leqq 1, \\
& \leqq 2 / 3 a, & 1 \leqq a \leqq 2 .
\end{array}
$$

Proof. The estimate $d^{*} \leqq 2 / 3 a$ for $1 \leqq a \leqq 2$ is proven by Netanyahu, see [6]. We will show $d^{*} \leqq 2 /(a+2)$ for $0 \leqq a \leqq 1$. Let $g(w)=w+c_{2} w^{2}+\cdots$ denote the inverse function to $w=f(z)$. A direct computation shows $c_{2}=-a$. Consider the function

$$
h(\zeta)=\frac{1}{d^{*}} \frac{g\left(d^{*} \zeta\right)}{\left(1+g\left(d^{*} \zeta\right)\right)^{2}}
$$

Since the composition of univalent functions is univalent and $h^{\prime}(0)=1, h(\zeta) \in U$. The second coefficient of $h(\zeta)$ is $-d^{*}(2+a)$. By [2], we have $d^{*}(2+a) \leqq 2$.

Applying Theorem 7 to Theorem 2 when $\alpha=0$, Lemma 5 gives the following lower bound for $d_{0} / d^{*}$.

LEMMA 6. If $w=f(w) \in \mathrm{St}$, then

$$
\begin{aligned}
d_{0} / d^{*} & \geqq \frac{a+2}{2}\left[\frac{r_{0}(a)}{1+a r_{0}(a)+r_{0}^{2}(a)}\right], & 0 \leqq a \leqq 1, \\
& \geqq \frac{3}{2} a\left[\frac{r_{0}(a)}{1+a r_{0}(a)+r_{0}^{2}(a)}\right], & 1 \leqq a \leqq 2 .
\end{aligned}
$$

In order to minimize the right-hand side of (29), the following lemma is needed. 
LEMMA 7. The function

$$
r_{0}(a)=\frac{a+\sqrt{ }\left(a^{2}+32\right)-\sqrt{ }\left[2 a^{2}+2 a \sqrt{ }\left(a^{2}+32\right)+16\right]}{4}
$$

is monotone decreasing for $0 \leqq a \leqq 2$.

Proof. By (27), $r_{0}(a)$ satisfies the equation

$$
1-a r_{0}(a)-6 r_{0}^{2}(a)-a r_{0}^{3}(a)+r_{0}^{4}(a)=0 .
$$

Using implicit differentiation, we have

$$
r_{0}^{\prime}(a)=\frac{r_{0}(a)\left[r_{0}^{2}(a)+1\right]}{4 r_{0}^{3}(a)-3 a r_{0}^{2}(a)-12 r_{0}(a)-a}
$$

which has a positive numerator. As for the denominator, we have

$$
4 r_{0}^{3}(a)-3 a r_{0}^{2}(a)-12 r_{0}(a)-a \leqq 4 r_{0}(a)\left(r_{0}^{2}(a)-3\right)<0
$$

because $0 \leqq a \leqq 2$ and $0<r_{0}(a)<1$. Therefore, $r_{0}^{\prime}(a)<0$ and $r_{0}(a)$ decreases.

THEOREM 5. If $w=f(z) \in \mathrm{St}$, then $d_{0} / d^{*} \geqq .343$...

Proof. If $0 \leqq a \leqq 1$, we have

$$
d_{0} / d^{*} \geqq \frac{a+2}{2}\left[\frac{r_{0}(a)}{1+a r_{0}(a)+r_{0}^{2}(a)}\right]=\frac{h(a)}{2} .
$$

By (27) we have

$$
a=\frac{1-6 r_{0}^{2}(a)+r_{0}^{4}(a)}{r_{0}(a)\left(1+r_{0}^{2}(a)\right)}
$$

Applying this equation to the function $h(a)$, we obtain

$$
\begin{aligned}
h(a) & =\frac{1}{2}\left[\frac{\left(1-r_{0}(a)\right)^{2}\left(1+4 r_{0}(a)+r_{0}^{2}(a)\right)}{\left(1-r_{0}(a)\right)^{2}\left(1+r_{0}(a)\right)^{2}}\right] \\
& =\frac{1}{2}\left[1+\frac{2 r_{0}(a)}{\left(1+r_{0}(a)\right)^{2}}\right] .
\end{aligned}
$$

Hence,

$$
h^{\prime}(a)=\left[\frac{1-r_{0}(a)}{\left(1+r_{0}(a)\right)^{3}}\right] r_{0}^{\prime}(a)
$$

Therefore, $h^{\prime}(a)<0$ because $r_{0}^{\prime}(a)<0$ and $0<r_{0}(a)<1$. From this we obtain $d_{0} / d^{*}$ $\geqq h(a) / 2 \geqq h(1) / 2$ when $0 \leqq a \leqq 1$.

If $1 \leqq a \leqq 2$, we have

$$
d_{0} / d^{*} \geqq \frac{3}{2} a\left[\frac{r_{0}(a)}{1+a r_{0}(a)+r_{0}^{2}(a)}\right]=\frac{3}{2} k(a)
$$

By (27) we have

$$
a r_{0}(a)=\frac{1-6 r_{0}^{2}(a)+r_{0}^{4}(a)}{\left(1+r_{0}^{2}(a)\right)}
$$


Substituting this equation into the function $k(a)$ we obtain

$$
k(a)=\frac{1}{2}\left[\frac{1-6 r_{0}^{2}(a)+r_{0}^{4}(a)}{\left(1-r_{0}^{2}(a)\right)^{2}}\right]
$$

Since

$$
k^{\prime}(a)=-4 r_{0}(a) r_{0}^{\prime}(a)\left[\frac{1+r_{0}^{2}(a)}{\left(1-r_{0}^{2}(a)\right)^{3}}\right]
$$

$r_{0}^{\prime}(a)<0$ gives $k^{\prime}(a)>0$. From this we obtain

$$
d_{0} / d^{*} \geqq \frac{3}{2} k(a) \geqq \frac{3}{2} k(1)
$$

when $1 \leqq a \leqq 2$. Thus we obtain the following

$$
d_{0} / d^{*} \geqq \frac{1}{2} h(1)=\frac{3}{2} k(1)=.343 \ldots
$$

6. Functions with $p$-fold rotational symmetry. Let $\mathrm{St}_{p}$ denote the class of functions $w=f(z)=z+\sum_{n=1}^{\infty} a_{n p+1} z^{n p+1} \in \mathrm{St}$ which have $p$-fold rotational symmetry. Using methods similar to those used in the previous sections, the following estimates may be proven for this class of functions where $a=a_{p+1} \geqq 0$,

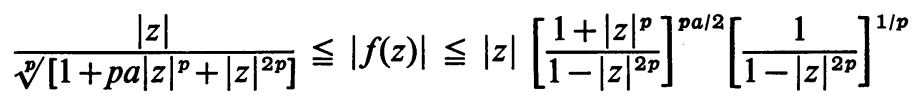

$$
\begin{aligned}
& r_{0} \geqq\left[\frac{p^{2} a+\sqrt{ }\left[p^{4} a^{2}+16(1+p)\right]-\sqrt{ }\left[2 p^{4} a^{2}+2 p^{2} a \sqrt{ }\left(p^{4} a^{2}+16+16 p\right)+16 p\right]}{4}\right]^{1 / p} \\
& d^{*} \leqq\left[\frac{2}{a p+2}\right]^{1 / p}, \quad 0 \leqq a \leqq \frac{1}{p} \\
& \leqq\left[\frac{2}{3 a p}\right]^{1 / p}, \quad \frac{1}{p} \leqq a \leqq \frac{2}{p}
\end{aligned}
$$

From these inequalities we obtain

THEOREM 7. If $w=f(z) \in \mathrm{St}_{p}$, then $d_{0} / d^{*}>2 / 3$ for $p \geqq 5$.

ACKNOWLEDGEMENT. The author wishes to thank the referee for his suggestions.

\section{BIBLIOGRAPHY}

1. M. Finkelstein, Growth estimates of convex functions, Proc. Amer. Math. Soc. 18 (1967), 412-418. MR 35 \#5598.

2. G. M. Golusin, Interior problems of the theory of schlicht functions, Uspehi Mat. Nauk 6 (1939), 26-89; English transl., Office of Naval Res. Navy Dept., Washington, D. C., 1947. MR 1, 49; MR 8, 575.

3. E. Gray and A. Schild, A new proof of a conjecture of Schild, Proc. Amer. Math. Soc. 16 (1965), 76-77. MR 30 \#2136.

4. T. H. Gronwall, On the distortion in conformal mapping when the second coefficient in the mapping function has an assigned value, Proc. Nat. Acad. Sci. U. S. A. 6 (1920), 300-302.

5. Z. Nehari, Conformal mapping, McGraw-Hill, New York, 1952. MR 13, 640. 
6. E. Netanyahu, The minimal distance of the image boundary from the origin and the second coefficient of a univalent function in $|z|<1$, Arch. Rational Mech. Anal. 32 (1969), 100-112. MR 38 \#3422.

7. E. Pflanz, Uber P-fach Symmetriche schlichte Functionen, Math. Z. 40 (1935), 72-85.

8. M. S. Robertson, On the theory of univalent functions, Ann. of Math. (2) 37 (1936), 374-408.

9. A. Schild, On a problem in conformal mapping of schlicht functions, Proc. Amer. Math. Soc. 4 (1953), 43-51. MR 14, 861.

10. - On a class of univalent star shaped mappings, Proc. Amer. Math. Soc. 9 (1958), 751-757. MR 20 \#2452.

11. - On a class of functions schlicht in the unit circle, Proc. Amer. Math. Soc. 5 (1954), 115-120. MR 15, 694.

12. - On starlike functions of order $\alpha$, Amer. J. Math. 87 (1965), 65-70.

13. E. Strohhäcker, Beitrage Zur Theorie der schlichten Functionen, Math. Z. 37 (1933), 356-380.

TeMPle UNIVERSITY,

Philadelphia, Pennsylvania 19122 